\title{
Tissue response to applied loading using different designs of penile compression clamps
}

This article was published in the following Dove Press journal:

Medical Devices: Evidence and Research

\author{
Joseph MH Lemmens' \\ Jackie Broadbridge' \\ Margaret Macaulay' \\ Rowland W Rees ${ }^{2}$ \\ Matt Archer ${ }^{2}$ \\ Marcus J Drake ${ }^{3}$ \\ Katherine N Moore ${ }^{4}$ \\ Dan L Bader' \\ Mandy Fader'
}

\begin{abstract}
'University of Southampton, School of Health Sciences, Southampton SOI7 IBJ, UK; ${ }^{2}$ Department of Urology, University Hospital Southampton NHS Foundation Trust, Southampton, SOI6 6YD, UK;

${ }^{3}$ Bristol Urological Institute, Southmead Hospital, Bristol, BSIO 5NB, UK; ${ }^{4}$ Faculty of Nursing, University of Alberta, Edmonton, Alberta, T6G IC9, Canada
\end{abstract}

Correspondence: Margaret Macaulay University of Southampton, School of Health Sciences, Level A, (MPII) South Academic Block, Southampton General Hospital, Tremona Rd., Southampton SOI6 6YD, UK

Tel: +4402381203919

Email m.macaulay@soton.ac.uk
Background: Penile compression devices (PCD) or clamps are applied to compress the urethra and prevent urinary incontinence (UI). PCDs are more secure and less likely to leak than pads, allowing men the opportunity to participate in short-term, vigorous activities. However, they are uncomfortable, can cause pressure ulcers (PU) and affect penile blood flow. No objective assessment of tissue health has been undertaken to assess and compare different PCD designs and to provide guidance on safe use.

Objective: This study was designed to evaluate existing PCDs in terms of their physiological response and potential for pressure-induced injury.

Design, setting and participants: Six men with post-prostatectomy UI tested four selected PCDs at effective pressures, in a random order, in a controlled laboratory setting.

Outcome measurements and statistical analysis: Using objective methods for assessing skin injury, PCDs were measured in situ for their effects on circulatory impedance, interface pressures and inflammatory response.

Results and limitations: There was evidence for PCD-induced circulatory impedance in most test conditions. Interface pressures varied considerably between both PCDs and participants, with a mean value of $137.4 \pm 69.7 \mathrm{mmHg}$. In some cases, penile skin was noted to be sensitive to loading with elevated concentration of the cytokine IL- $1 \alpha$ after 10 mins wear, indicating an inflammatory response. IL- $1 \alpha$ levels were restored to baseline 40 mins following PCD removal.

Conclusion: Skin health measures indicated tissue and blood flow compromise during the 50 mins of testing using all PCDs. Although there was an elevation in pro-inflammatory cytokines, PCDs did not cause sustained irritation and skin health measures recovered 40 mins after PCD removal. This research indicates that application of a clamp for one hour with an equal clamp free time before reapplication is likely to be safe. Longer periods are often recommended by manufacturers but have yet to be tested.

Keywords: penile, compression, clamp, urinary, incontinence, pressure

\section{Introduction}

Urinary incontinence (UI) is a complication suffered by men who undergo surgery for prostate cancer. This debilitating condition has a major effect on quality of life, with an impact considered greater than erectile dysfunction (ED), ${ }^{1}$ affecting personal and professional relationships, and can lead to depression and social isolation. ${ }^{2-5}$ Although the majority of men recover continence within 12 months, about $15 \%$ will be affected throughout their lifetime and require non-surgical methods to manage the leakage. ${ }^{6}$ Incontinence pads represent a common management choice but they have many drawbacks. They can be bulky, hot, difficult to dispose of, require considerable storage space and many men consider them to 
have an unacceptable "babyish or feminine" appearance. ${ }^{7}$ Sheaths, body-worn urinals (BWU) and PCDs are alternatives or adjuncts to pads. Of these, PCDs have been reported to be particularly useful for certain activities when a secure, discreet device is required. ${ }^{8}$ However, little has been published on the correct use or applied pressures necessary for these devices ${ }^{8,9}$ and if used improperly, PCDs can place the individual at risk for penile trauma. Such a lack of evidence on safety is a barrier to their use. Lack of prescribing means that appropriate candidates are prevented from using a potentially effective short-term continence option. Moreover, it may mean that men purchase PCDs online and use them without professional support and advice, with the potential risk of penile injury in the form of medical-device related pressure ulcers (MDRPU).

Only two studies have evaluated PCDs, in terms of penile blood flow and user acceptability. ${ }^{8,9}$ The first reported that the Cunningham PCD was the most effective of three PCDs at significantly reducing leakage, with an associated decrease in penile blood flow from 125 to $73 \mathrm{~mm} / \mathrm{s}^{9}{ }^{9}$ In the other larger study, the Cunningham PCD was also reported to be the most effective device at reducing leakage and being secure, but was more painful compared with non-compression products ie, sheaths, BWUs and pads. Those who could tolerate the PCD found it to be useful for short vigorous activities such as swimming or dancing. ${ }^{8}$ Although neither study reported on pressure-related injury, several case reports ${ }^{10,11}$ attest to the risk of mechanically induced penile soft tissue injury and the formation of pressure ulcers.

Currently, there is insufficient research on PCDs to allow evidence-based decisions by clinicians and consumers. It appears that current products are limited by discomfort and urine leakage as well as a potential for penile injury and reduced penile blood flow. There is a role for a safe, effective clamp to augment continence management, but increased understanding is required of the effects on penile perfusion and skin health for existing PCDs before users and HCPs can prescribe them with confidence.

This study represents part of a larger research programme to investigate the performance of PCDs. It utilizes established objective bioengineering techniques to assess the effects of applied loading on skin response incorporating four commercially available PCDs, which are applied at a pressure sufficient to restrict incontinence.

\section{Aims and objectives}

\section{Aim}

To evaluate the tissue response to different commercially available PCDs with a view to assessing potential risk of their use.

\section{Objectives}

To evaluate applied interface pressure, circulatory impedance and inflammatory response when PCDs are applied at relevant pressures necessary to reduce or eliminate UI in men post-prostatectomy.

\section{Materials and methods Participants}

Experienced PCD users with post-prostatectomy UI were recruited from previous participants, ${ }^{8}$ prostate cancer support groups and incontinence organizations. The inclusion criteria included men with UI for greater than 12 months after radical prostatectomy, who had experience of using a PCD. The exclusion criteria included the self-reported absence of sensation to the penis, urgency or urgency incontinence, ie, a strong uncontrollable urge to pass urine and leakage before reaching the toilet, as the predominant urinary symptom. In addition, men were excluded with a poor memory or forgetfulness (Mini Mental State Exam <27) leading to an inability to reliably release the PCD regularly at least every $2 \mathrm{hrs,} \mathrm{and}$ those in the terminal stages of an illness.

Ethical approval was given by the South Central Hampshire B Research Ethics Committee (14/SC/1034, 05.09.14) and informed written consent was obtained from each volunteer. The study was conducted in accordance with the Declaration of Helsinki.

\section{Devices}

Samples of PCDs available worldwide $(\mathrm{n}=14)$ were obtained. A group of experienced PCD users tried each PCD for 30 mins, while also wearing a pre-weighed pad, during a standard exercise regime. Leakage was assessed based on subtraction of the pre-test pad weight from the post-test pad weight. A subset of four PCDs was selected for laboratory testing representing the main designs, while being both the most effective at preventing urine loss in the pad test and the most acceptable to users (Figure 1).

\section{Participant testing and procedures}

As blinding was not possible, PCDs were tested in random order determined through use of Latin squares. ${ }^{12}$ A series of tests was performed for each PCD with each participant to 


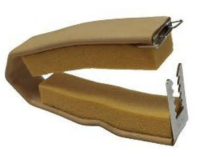

Cunningham, Bard Medical

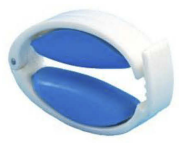

Wiesner,

Wiesner Healthcare Innovation LLC

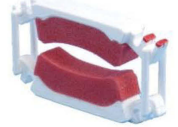

Dribblestop ${ }^{\circledR}$, Rennich Industries Ltd.

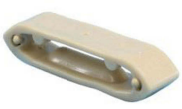

Uriclak $^{\circledR}$, Vitalnovax $\mathrm{Cb}$

Figure I PCDs selected for laboratory testing.

measure applied interface pressure, circulatory impedance and inflammatory response. After baseline measurements were recorded in the absence of a device in situ (Time 0), participants applied the PCD to a tightness which they judged from previous experience to be tolerable and effective, termed the prescribed interface pressure (PIP). Measurements were then recorded during wear 10 mins and 50 mins after application, immediately after removal, ie, 55 mins after application, and after a 40 mins recovery period, ie, 90 mins after application (Table 1).

\section{Test outcomes}

\section{Applied interface pressure}

Applied interface pressures between the PCD and the soft tissues of the penis were measured, using an established commercial system (Talley Mk3 Pressure Monitor, Talley Medical, UK). This electro-pneumatic based system has a reported mean error of $12 \pm 1 \% .^{13}$ The measurement involved positioning an individual air cell, $18 \mathrm{~mm}$ in diameter, on the ventral surface of the penis under the arms of the PCD (Figure 2).

\section{Circulatory impedance}

Laser Doppler perfusion imaging (LDPI) represents an established method for assessment of penile perfusion ${ }^{9}$ and circulatory impedance. A commercial system (LDI2-VR, Moor Instruments, UK), activated at a wavelength of $633 \mathrm{~nm}$ at a power of $2.5 \mathrm{~mW}$, was used to measure blood flux in arbitrary units (AU) at the dorsal surface of the penis within three regions of interest (ROI), namely, proximal, central and distal (Figure 2) to the PCD at a sub-dermal depth of approximately $0.6 \mathrm{~mm} .^{14}$

\section{Inflammatory response}

Sebutape $^{\circledR}$ (CuDerm Corporation, Dallas, Texas, USA) represents a hydrophobic lipid absorbent micro-porous film used to collect skin sebum. It has been previously employed to examine a number of pro-inflammatory cytokines, using a well-established protocol. ${ }^{15,16} \mathrm{~A}$ Sebutape sample, $400 \mathrm{~mm}^{2}$ in area, was placed on the dorsal side of the penis between the PCD and the skin and the PCD was applied at the PIP for 2 mins (Figure 2). Samples were then carefully removed using blunt forceps and placed in plastic low-bind $2 \mathrm{~mL}$ microtubes (Axygen ${ }^{\mathrm{TM}}$ MaxyClear), which were then placed on ice and frozen at $-80^{\circ} \mathrm{C}$. Collections using fresh Sebutape were repeated throughout PCD wear and recovery periods (Table 1).

\section{Biochemical analysis}

Frozen Sebutapes ${ }^{\circledR}$ were rapidly thawed to room temperature and $1.7 \mathrm{~mL}$ of phosphate buffered saline (PBS; Sigma-Aldrich Co., St Louis, US) solution was added to each vial. After $1 \mathrm{hr}$, the tapes were sonicated for $10 \mathrm{mins}$ at $20^{\circ} \mathrm{C}$, vortexed vigorously for $2 \mathrm{mins}$ and additionally mixed with a pipette tip. After refreezing overnight at $-80^{\circ} \mathrm{C}$, the tape extracts were thawed, vortexed for $1 \mathrm{~min}$ and mixed with a pipette to recover the total extracts from the tapes. Extracts were aliquoted into five vials (Thermo Scientific ${ }^{\mathrm{TM}}$ Low

Table I Laboratory testing timeline for measurements

\begin{tabular}{|l|l|l|l|l|l|l|}
\hline \multirow{2}{*}{ Timeline (minutes) $\rightarrow$} & \multirow{2}{*}{ Baseline (no PCD) } & \multicolumn{2}{|l|}{ PCD applied ("wear time” =50 mins) } & \multicolumn{2}{l|}{ PCD removed ("rest time” =40 mins) } \\
\cline { 3 - 7 } & & 0 & 10 & 50 & 55 & 90 \\
\hline Test $\rightarrow$ & Cl; IR & IP & Cl; IR; IP & Cl; IR; IP & Cl & Cl; IR \\
\hline
\end{tabular}

Abbreviations: $\mathrm{PCD}$, penile compression device; $\mathrm{Cl}$, circulatory impedance; IR, inflammatory response; IP, interface pressure. 


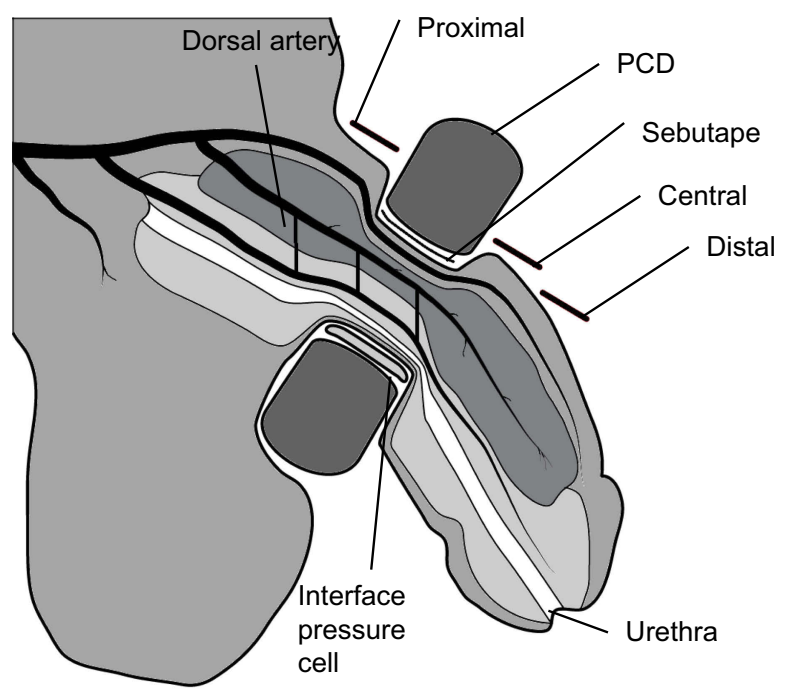

Figure 2 Location of the three measurements to assess the skin response on the penis when subjected to each of the penile compression devices (PCD) applied at their associated prescribed interface pressures.

Retention). Samples from all participants were then processed and analyzed in triplicate using immunoassay kits (PeproTech ELISA ABTS kits, New Jersey, USA) to estimate concentrations of IL- $1 \alpha$ with a detection range of 8-1,000 $\mathrm{pg} / \mathrm{mL}$. Sample dilutions ensured the detection was extended up to $4,000 \mathrm{pg} / \mathrm{mL}$.

\section{Data analysis}

Descriptive and inferential statistics were performed using Microsoft Excel. Non-parametric descriptors were used for cytokine concentrations. Due to the small size, a Wilcoxon Rank Sum Test was used for paired cytokine measures and to evaluate the effects of clamping pressure on blood perfusion flux. Statistical significance was defined as $p<0.05$.

\section{Results}

\section{Participants}

Six experienced clamp users were recruited into the study, with a mean age of $71.5 \pm 4.97$ years and a mean BMI of $26.45 \pm 3.14 \mathrm{~kg} / \mathrm{m}^{2}$.

\section{Interface pressure}

PIP varied considerably with a mean value of $137.4 \pm 69.7$ $\mathrm{mmHg}$, thus precluding any inter-participant comparisons. Figure 3 shows the mean interface pressures measured at 0, 10 and 50 mins during the wear period for each PCD and participant. In many cases, there was a moderate variation in interface pressure values over the $50 \mathrm{mins}$ wear period. It is noteworthy that there was a decrease between 10 and 50 mins wear for the Dribblestop PCD (Figure 3B and 3E), compared to a corresponding temporal increase for the Uriclak PCD (Figure 3D and 3F).
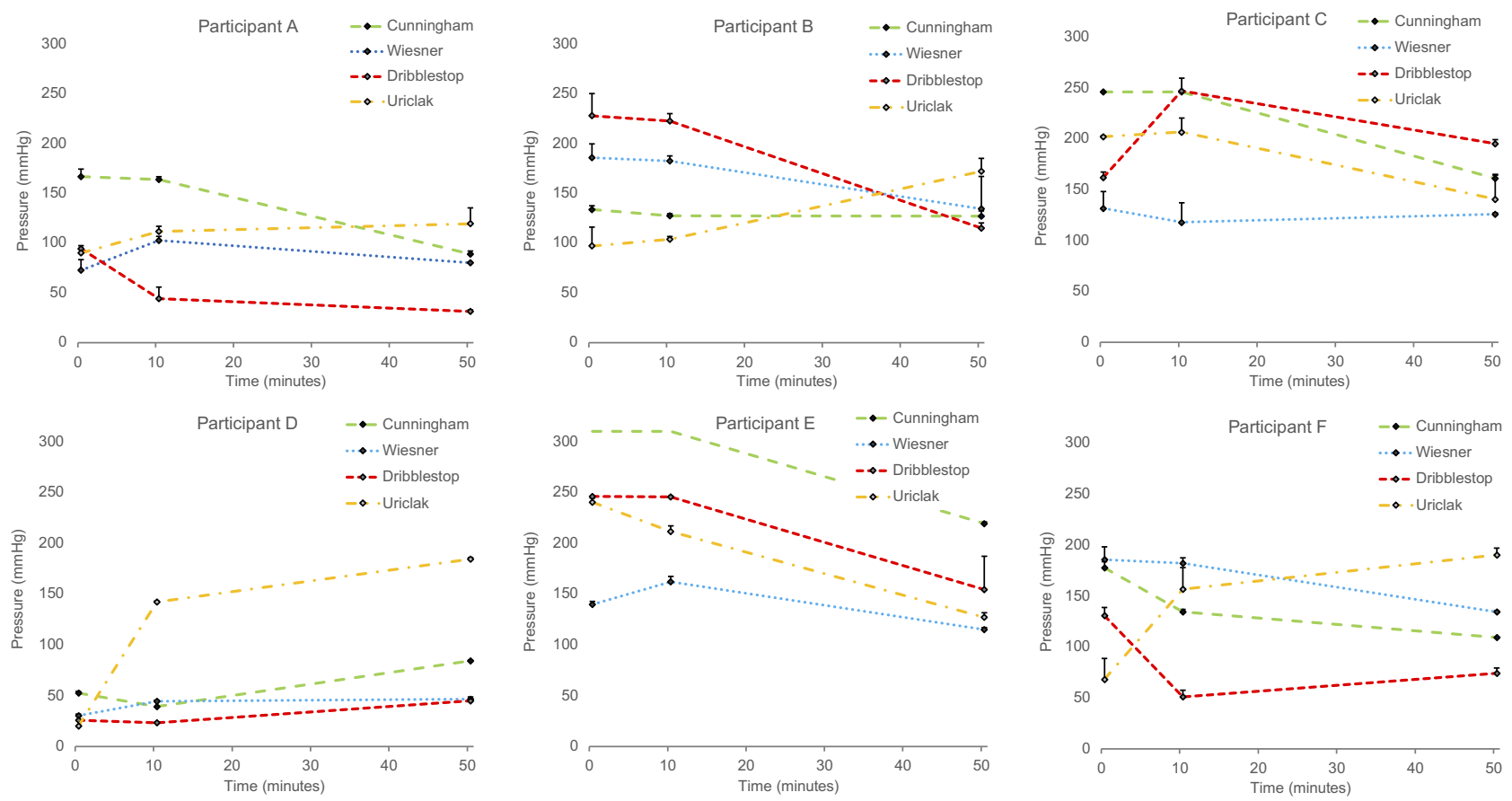

Figure 3 Temporal profiles of mean interface pressure values \pm SD for six participants and each of the penile compression devices ( PCDs) over the 50 mins wear period. 


\section{Circulatory impedance}

There was a small change in perfusion for each PCD over the 50 mins wear period. This is illustrated with two of the participants in Figure 4. The Cunningham PCD caused the most significant decrease in blood perfusion, with values below 50 AUs in both central and distal ROIs. Figure 5 illustrates the decrease with the Dribblestop PCD. The effect of removing the PCDs was estimated by examining the perfusion values during the first 5 mins of the recovery period. It is evident that removal of three of the PCDs (Cunningham, Uriclak and Dribblestop) resulted in an increased perfusion, indicating a reactive hyperemic response following circularity occlusion. By contrast, the Wiesner PCD exhibited only small changes during both the wear and recovery periods.
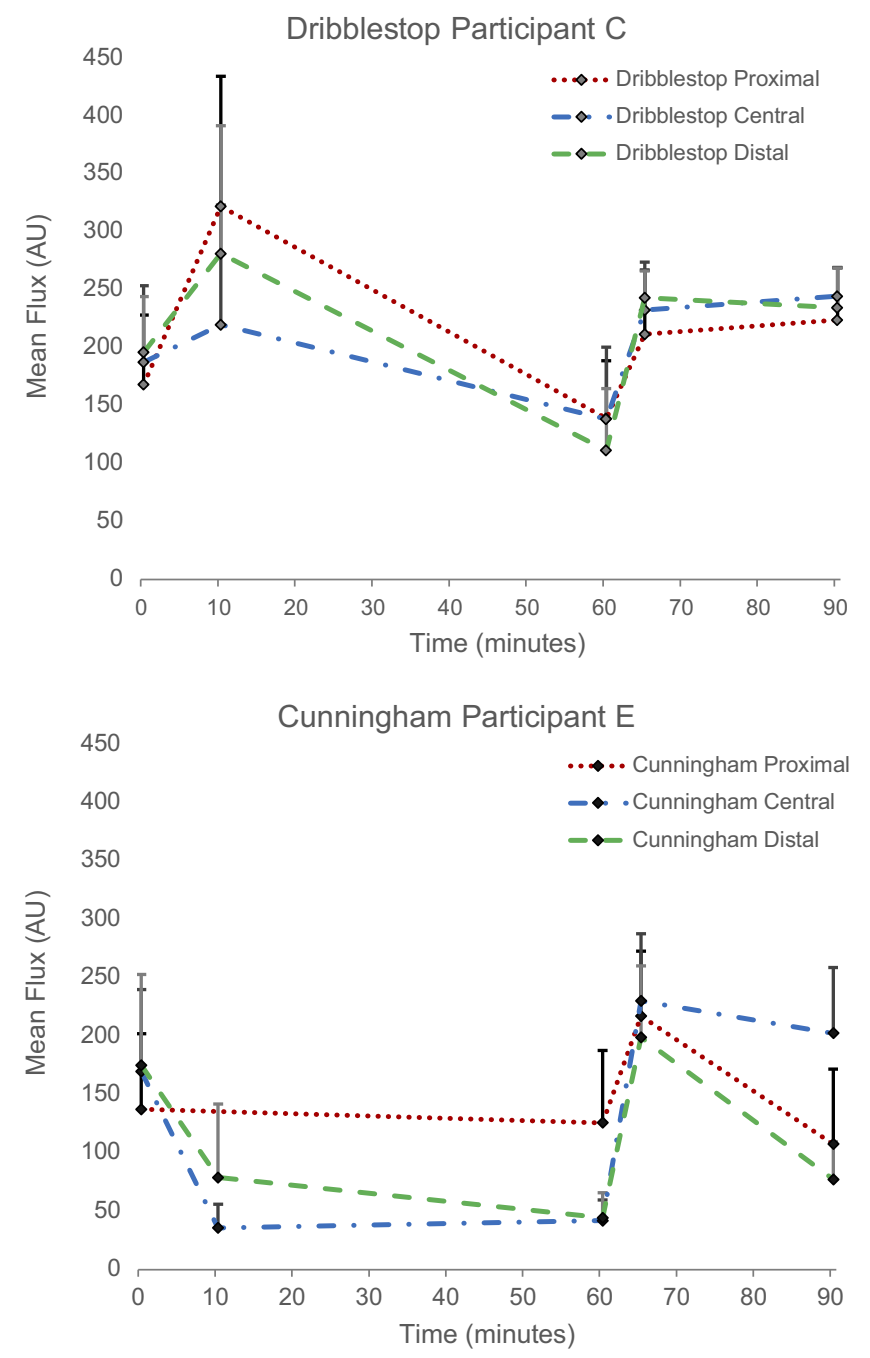

There were noticeable differences in perfusion values between the different ROIs. For example, by assuming unequal variances, a statistically significant difference was found between pressure and mean perfusion flux within the central ROI $(p \leq 0.01)$. In addition, there was a statistically significant difference between values at baseline and after 10 mins of wear in the distal ROI $(p \leq 0.01)$ alone.

Following release of the PCD at the end of the wear period, there was a significant difference in perfusion at the proximal ROI only $(p<0.05)$. No significant differences were evident between the end of the recovery period and baseline values for all three ROIs, suggesting that flow returns to normal after a $40 \mathrm{mins}$ recovery period.

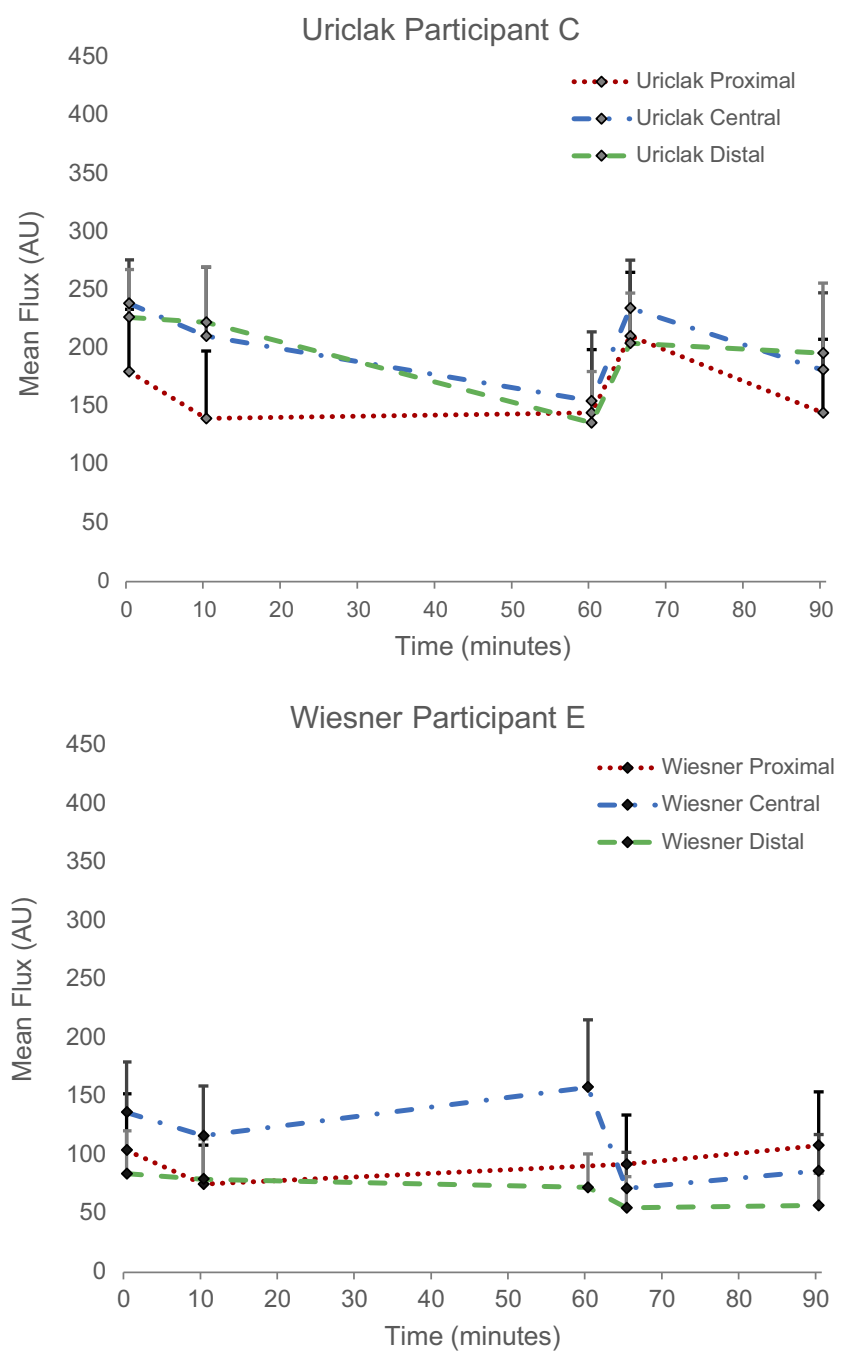

Figure 4 Temporal profiles of Doppler ROI values in three regions of the penis of two participants for each of the penile compression device (PCD) designs. Error bars represent perfusion flux SD 
A
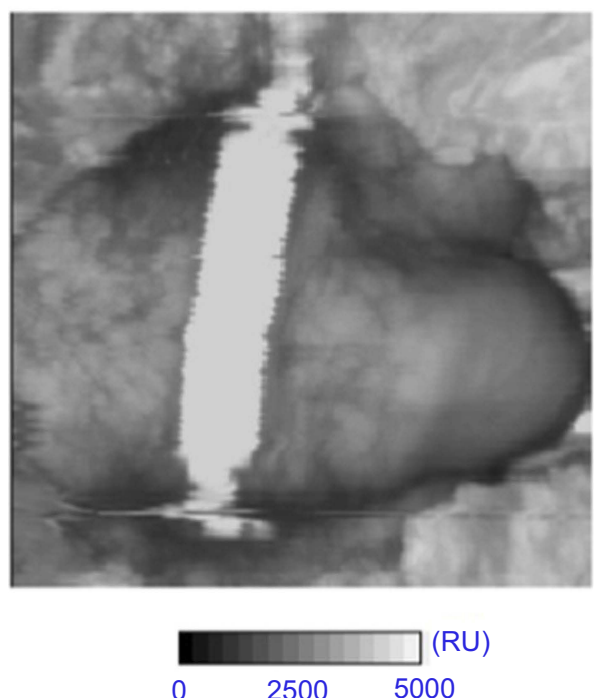

B

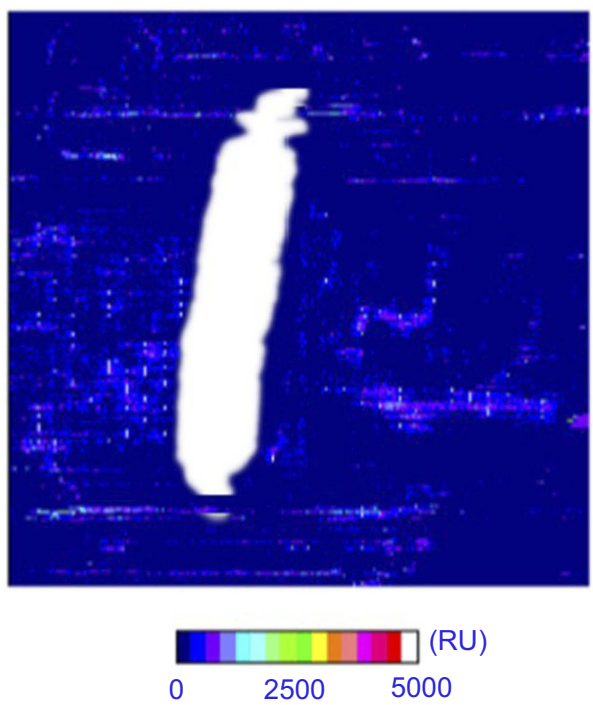

Figure 5 The Dribblestop penile compression device (PCD) had, by 60 mins wear time, decreased distal blood perfusion flux (Figure 4) after an initial rise corresponding to a sustained increase in interfacial pressure (Figure 3). (A) Scanned image (B) PCD location superimposed over flux scan.

\section{Inflammatory response}

Figure 6 reveals considerable intra-individual variability in IL-1 $\alpha$ concentrations. Nonetheless, four of the participants (A, B, C and D) produced similar IL- $1 \alpha$ concentration profiles with a general increase over baseline at $10 \mathrm{mins}$, which was maintained throughout the wear period. Following the 40 mins recovery period, cytokine levels had returned to baseline, resulting in no significant differences between their values and those of baseline $(p>\alpha=0.05)$.

Although participant E, who presented with a significant penile retraction, reported a preference for the Uriclak PCD, this design yielded a higher release of IL- $1 \alpha$ than the other PCDs. The Cunningham PCD was applied at the
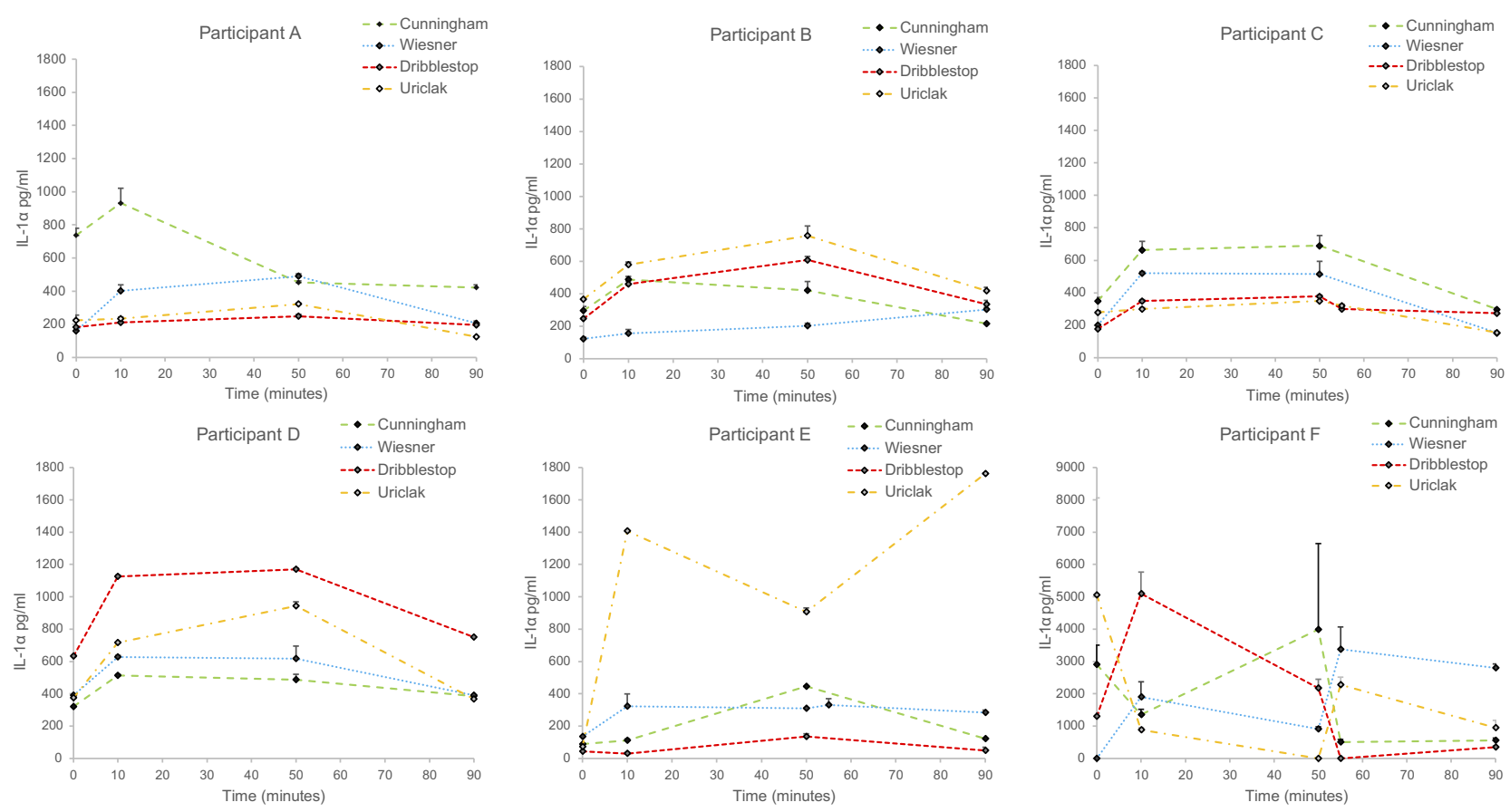

Figure 6 Inflammatory response (IL-l $\alpha$ [pg/mL]) measured at the skin surface over the 90 mins test period. 
highest pressures in excess of $250 \mathrm{mmHg}$ (Figure 3E). Immediately after removal, an IL- $1 \alpha$ concentration value beyond the limit of the IL-1 detection was obtained.

\section{Discussion}

This study was designed to evaluate four existing PCDs for penile physiological response and potential for pressure-induced injury. With pressures adequate to prevent UI in male volunteers, we found reduced circulatory impedance and raised superficial inflammatory response, as reflected in an up-regulation of IL- $1 \alpha$. These raised levels indicate a potential for skin and soft tissue injury depending on wear time and applied pressure and suggest that design improvements are warranted. We did not find that overall any one design produced a less adverse impact on physiological measurements than the others.

A computer model confirms the limitations of current PCD designs and the potential risk of soft tissue damage. ${ }^{17}$ Interface pressure of $188 \mathrm{mmHg}$ within the range of the present in vivo study (Figure 3) caused internal stresses greater than $75 \mathrm{mmHg}$ in the skin, fat and tunica albuginea proximal to the urethra. Adjusting the model to reflect slight changes in the angle of the PCD, as would occur in normal wear, resulted in an increase in tissue strains/ deformations and tissue stresses. The highest stress on the urethra was predicted with the Wiesner PCD, a design which utilizes a rigid knurl behind a silicone cover (Figure 1). In our study, the Wiesner did not generate significantly different pressures or circulatory impedance when compared to the other designs but when it was trialed at home, men reported more discomfort and one incident of haematuria. ${ }^{18}$

Each PCD restricted venous return to some extent resulting in blood pooling distally and engorgement of the penis. However, three of the PCDs (Dribblestop, Cunningham and Wiesner) loosened during wear and required adjustment as evidenced by the small reduction in interface pressures. This may be a result of various factors including the viscoelastic behavior of soft tissues, ${ }^{19}$ softening of the erectile state and possible reduction in the flexural modulus of the device materials due to body heat and moisture. By contrast, the Uriclak PCD, which operates using a sprung metal section (Figure 3D), did not loosen with wear and its continued use resulted in increased interface pressures in four of the six participants.

Compression of blood vessels by the PCDs affected penile circulation as measured by Doppler perfusion flux (Figure 5). Importantly, on removal, perfusion levels were restored to unloaded levels after the 40 mins recovery period.
Although three of the participants (Figure 3: B, C, E) recorded pressures in excess of $250 \mathrm{mmHg}$, the corresponding mean perfusion values were also high (Figure 4). This anomaly might be explained by compression causing partial constriction of a number of blood vessels within the network with a corresponding increased flow within the remaining vessels. However, this finding could still indicate total underperfusion in the vulnerable penile tissues, as the network of capillaries is copious and there may be hypoxia within the deep tissues of the corpora cavernosa.

Potential pressure injury to the penis from impaired circulation has been studied in cyclists. Compression by bike saddles has been associated with perineal and penile trauma including neural injury, erectile dysfunction and numbness. In one small cohort study, MR images revealed large mean compressive strains of $56 \%$ and $67 \%$ in the corpus spongiosum and the corpus cavernosum, respectively. ${ }^{20}$ These strains were associated with mean anterior and posterior saddle pressures of $110 \mathrm{mmHg}$ and $146 \mathrm{mmHg}$, respectively, values similar in magnitude to those measured in the present study.

As pressures from the PCD may cause ischemia, there could be cellular damage from a reduction in tissue oxygen supply. Our study did not measure oxygen levels directly, but found evidence of reactive hyperemia. In another type of penile device, a vacuum compression device for erectile dysfunction, there have been no reports of persistent penile injury where a marked reperfusion hyperemia could be anticipated. $^{21}$ Further research is required to assess the cumulative effect on tissues of continued PCD use.

The inflammatory response to applied compression was assessed by the up-regulation of the primary cytokine, IL$1 \alpha$, which is secreted by keratinocytes in response to skin inflammation. IL- $1 \alpha$ was rapidly released at the surface of the stratum corneum and collected in the sebum. Although a degree of inter- and intra-participant variability was evident, the trends in the IL- $1 \alpha$ secretion profiles were similar (Figure 6). Increased IL-1 $\alpha$ up-regulation was evident from baseline to 10 mins, ie, immediately after PCD application, plateauing by 50 mins coincident with two of the PCDs, the Uriclak and, to a lesser extent, the Dribblestop, resulting in an increased pressure over wear time (Figure 3). It is noteworthy that penile skin is particularly sensitive to mechanical loading as reflected in high concentrations of IL- $1 \alpha$ in sebum when compared to that released from facial or forearm skin. ${ }^{22}$ No significant differences were seen between baseline levels and the values of IL-1 $\alpha$ after a 40 mins recovery period. 
For practical reasons, the test protocol was limited to a wear period of 50 mins. It is accepted that in normal use, individuals are likely to leave the PCD in situ for longer periods. Extended wear times might result in the development of superficial pressure ulcers (SPU) caused by elevated interface pressures, in conjunction with adverse microclimate conditions, typically, elevated skin surface temperature, humidity and air movement ${ }^{23}$ and it is, for example, well established that moisture at the device-skin interface increases friction forces. ${ }^{24}$

The study only included six participants. Considerable variability occurred between both individuals and the different PCDs. This is likely to be a reflection of several factors including the different PCD designs, the variability of male anatomy, skin sensitivity and the viscoelastic behavior of soft tissues. ${ }^{19}$ Despite this small number of and high variability between and within participants, objective methods that demonstrated physiological response to a relatively short period of PCD wear were validated. Whether a pressure ulcer would form with extended wear times remains unknown, ${ }^{25}$ due to the lack of published data for penile skin and the short wear period observed in this study.

\section{Conclusion}

PCDs have proved as useful medical devices for some men who suffer from urinary incontinence following prostatectomy. Little safety research exists to assure men and healthcare professionals that these devices are safe to use. In this study, tissue and blood flow were compromised and pro-inflammatory cytokines raised during the wear time of 50 mins, when a PCD was applied at a pressure adequate to maintain continence during brief activities. However, none of the PCDs caused sustained irritation or impaired blood flow and yielded good recovery 40 mins after their removal. An important question remains as to how long individuals can safely wear a PCD and, after removal, how soon it can be re-applied.

This research indicates that application of a clamp for one hour with an equal clamp free time before reapplication is likely to be safe. Longer periods are often recommended by manufacturers but have yet to be tested.

\section{Acknowledgments}

This work was funded by the Movember Foundation in partnership with Prostate Cancer UK as part of True NTH programme. Grant reference 250-10 Continence
Management. Support was also provided by the EPSRCNIHR Network Plus Award (EP/NO2723X/1) "Medical Devices and Vulnerable Skin: Intelligent sensing to promote self-management". The research protocol was registered in the www.public-odp.nihr.ac.uk database as number CPMS ID 17534. URL: http://www.goo.gl/ hPTYg8.

\section{Disclosure}

The following authors are named on a patent relating to the development of a new penile compression device - M Fader, DL Bader, JMH Lemmens, J Broadbridge, M Macaulay, R Rees, MJ Drake. MJ Drake reports personal fees, non-financial support from Astellas, grants from Ferring, non-financial support from Allergan. M Fader reports grants from Prostate Cancer UK (charity), during the conduct of the study. R Rees is a trainer for Boston Scientific and Coloplast. The authors report no other conflicts of interest in this work.

\section{References}

1. Mazur DJ, Merz JF. Older patients' willingness to trade off urologic adverse outcomes for a better chance at five-year survival in the clinical setting of prostate cancer. J Am Geriatr Soc. 1995;43:979984.

2. Kirschner-Hermanns R, Jakse G. Quality of life following radical prostatectomy. Crit Rev Oncol Hematol. 2002;43:141-151. doi:10.1016/S1040-8428(02)00026-4

3. Sharpley CF, Bitsika V, Christie DRH. Understanding the causes of depression among prostate cancer patients: development of the effects of prostate cancer on lifestyle questionnaire. Psychooncology. 2009;18:162-168. doi:10.1002/pon.1382

4. Resendes LA, McCorkle R. Spousal responses to prostate cancer: an integrative review. Cancer Invest. 2006;24:192-198. doi:10.1080/ 07357900500524652

5. Paterson J. Stigma associated with postprostatectomy urinary incontinence. J Wound Ostomy Cont Nurs. 2000;27:168-173.

6. Hunskaar S, Burgio K, Clark A, Lapitan M, Nelson R, Sillen U. Epidemiology of Urinary (UI) and Faecal (FI) incontinence and Pelvic Organ Prolapse (POP). In: Abrams P, Cardozo L, Khoury S, Wein A, editors. Incontinence (3rd Int. Con.). Health Publications Ltd., Jersey; 2005:255-312.

7. Cottenden A, Bliss D, Fader M, et al. Management with continence products. In: Abrams P, Cardozo L, Khoury S, Wein A, editors. Incontinence (3rd Int. Con.). Health Publications Ltd., Jersey; 2005. 149-253.

8. Macaulay M, Broadbridge J, Gage H, et al. A trial of devices for urinary incontinence after treatment for prostate cancer. BJU Int. 2015;116:432-442. doi:10.1111/bju.13016

9. Moore KN, Schieman S, Ackerman T, Dzus HY, Metcalfe JB, Voaklander DC. Assessing comfort, safety, and patient satisfaction with three commonly used penile compression devices. Urology. 2004;63:150-154. doi:10.1016/j.urology.2003.08.034

10. Carls C, Ermer-Seltun J, Rolstad BS. Partial thickness wound resulting from use of a penile clamp to control urinary incontinence in a patient with radical prostatectomy. Poster Symp Adv Wound Care. 2005. 
11. Kalra S, Srinivas PR, Manikandan R. Urethral diverticulum: a potential hazard of penile clamp application for male urinary incontinence. BMJ Case Rep. 2015;1-2. doi:10.1136/bcr-2015-209957

12. Armitage P, Berry G, Matthews JNS. Sec. 9.4 Latin Squares. Stat Methods Med. Res., Blackwell Scientific Publications. 2013.

13. Allen V, Ryan DW, Lomax N, Murray A. Accuracy of interface pressure measurement systems. J Biomed Eng. 1993;15:344-348.

14. Ash C, Dubec M, Donne K, Bashford T. Effect of wavelength and beam width on penetration in light-tissue interaction using computational methods. Lasers Med Sci. 2017;32:1909-1918. doi:10.1007/ s10103-017-2317-4

15. Perkins MA, Osterhues MA, Farage MA, Robinson MK. A noninvasive method to assess skin irritation and compromised skin conditions using simple tape adsorption of molecular markers of inflammation. Skin Res Technol. 2001;7:227-237. doi:10.1034/j.1600-0846.2001.70405.x

16. Perkins MA, Cardin CW, Osterhues MA, Robinson MK. A non-invasive tape absorption method for recovery of inflammatory mediators to differentiate normal from compromised scalp conditions. Skin Res Technol. 2002;8:187-193. doi:10.1034/j.1600-0846.2002.20337.x

17. Levy A, Fader M, Bader D, Gefen A. Penile compression clamps: a model of the internal mechanical state of penile soft tissues. Neurourol Urodyn. 2016;1-6. doi:10.1002/nau.23172

18. Lemmens JMH, Broadbridge J, Macaulay M, et al. Physiological impact and user acceptability of penile compression devices (PCD). Int Cont Soc Annu Mtg. 2017;36:155.Florence Italy 2017 Abstr 83 Neurourol Urodynam. doi:10.1002/nau.23386
19. Mayrovitz HN, Sims N. Biophysical effects of water and synthetic urine on skin. Adv Skin Wound Care. 2001;14:302-308. doi:10.1097/ 00129334-200111000-00013

20. Bressel E, Reeve T, Parker D, Cronin J. Influence of bicycle seat pressure on compression of the perineum: a MRI analysis. J Biomech. 2007;40:198-202. doi:10.1016/j.jbiomech.2005.11.017

21. Yuan J, Hoang AN, Romero CA, Lin H, Dai Y, Wang R. Vacuum therapy in erectile dysfunction - science and clinical evidence. Int $J$ Impot Res. 2010;22:211-219. doi:10.1038/ijir.2010.4

22. Worsley PR, Prudden G, Gower G, Bader DL. Investigating the effects of strap tension during non-invasive ventilation mask application: a combined biomechanical and biomarker approach. Med Devices Evid Res. 2016;9:409-417. doi:10.2147/MDER. S121712

23. Baharestani M. et al. International review. Pressure ulcer prevention: pressure, shear, friction and microclimate in context. A consensus document. London: Wounds International, 2010P;1-25.

24. Gefen A. How do microclimate factors affect the risk for superficial pressure ulcers: a mathematical modeling study. J Tissue Viability. 2011;20:81-88. doi:10.1016/j.jtv.2010.10.002

25. Beeckman D, Schoonhoven L, Fletcher J, et al. EPUAP classification system for pressure ulcers: European reliability study. J Adv Nurs. 2007;60:682-691. doi:10.1111/j.1365-2648.2007.04474.x
Medical Devices: Evidence and Research

\section{Publish your work in this journal}

Medical Devices: Evidence and Research is an international, peerreviewed, open access journal that focuses on the evidence, technology, research, and expert opinion supporting the use and application of medical devices in the diagnosis, monitoring, treatment and management of clinical conditions and physiological processes. The identification of novel devices and optimal use of existing devices which will lead to improved clinical outcomes and more effective patient management and safety is a key feature of the journal. The manuscript management system is completely online and includes a very quick and fair peer-review system. Visit http:// www.dovepress.com/testimonials.php to read real quotes from published authors. 